\title{
SÍNDROME DE BURNOUT ENTRE ENFERMEIROS DE UM HOSPITAL UNIVERSITÁRIO
}

\author{
BURNOUT SYNDROME AMONG NURSES \\ IN A UNIVERSITY HOSPITAL
}

\section{SÍNDROME DE BURNOUT ENTRE ENFERMEROS DE UN HOSPITAL UNIVERSITARIO}

\author{
José Luís Guedes dos Santos ${ }^{1}$ \\ Rosângela Marion da Silva ${ }^{2}$ \\ Caroline Cechinel Peiter ${ }^{3}$ \\ Fernando Henrique Antunes Menegon ${ }^{4}$ \\ Alacoque Lorenzini Erdmann ${ }^{5}$
}

Como citar este artigo: Santos JLG, Silva RM, Peiter CC, Menegon FHA, Erdmann AL. Síndrome de burnout entre enfermeiros de um hospital universitário. Rev baiana enferm. 2019;33:e29057.

Objetivo: identificar os níveis da síndrome de burnout entre enfermeiros de um hospital universitário. Método: estudo transversal, realizado em um hospital universitário da Região Sul do Brasil, com 106 enfermeiros. Os dados foram coletados por meio de um questionário composto por dois instrumentos autorrespondidos: ficha de caracterização pessoal e profissional e Inventário de Burnout de Maslach. Resultados: os enfermeiros participantes da pesquisa apresentaram níveis moderados de burnout para as três subescalas: exaustão emocional $(21,9 \pm 5,0)$, despersonalização $(8,1 \pm 2,6)$ e realização pessoal $(30,4 \pm 3,2)$. Enfermeiros do centro cirúrgico apresentaram índice alto na subescala exaustão emocional. Conclusão: identificou-se um nível moderado de burnout entre os enfermeiros de um hospital universitário para as três subescalas - exaustão emocional, despersonalização e realização pessoal. Não foram identificadas diferenças significativas entre as médias de burnout e as características socioprofissionais dos participantes, nem entre os setores hospitalares

Descritores: Esgotamento Profissional. Satisfação no Emprego. Estresse Psicológico. Enfermagem.

Objective: to identify burnout syndrome levels among nurses at a university hospital. Method: a cross-sectional study conducted in a university hospital in southern Brazil, with 106 nurses. Data was collected through a questionnaire composed of two self-answered instruments: personal and professional characterization form and the Maslach Burnout Inventory. Results: the nurses participating in the study presented moderate burnout levels for the

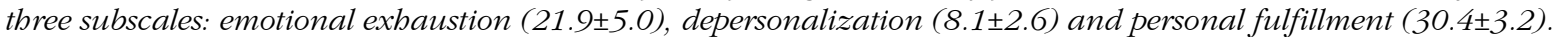
The nurses working in the surgical center had a bigh rate on the emotional exhaustion subscale. Conclusion: a moderate level of burnout was identified among the nurses working in a university hospital for the three subscales:

\footnotetext{
Enfermeiro. Doutor em Enfermagem. Professor Adjunto da Universidade Federal de Santa Catarina. Florianópolis. Santa Catarina, Brasil. jose.santos@ufsc.br. https:// orcid.org/0000-0003-3186-8286

2 Enfermeira. Doutora em Ciências. Professora Adjunta da Universidade Federal de Santa Maria. Santa Maria, Rio Grande do Sul, Brasil. https://orcid.org/0000-00033978-9654

Enfermeira. Mestre em Saúde Coletiva. Professora Substituta da Universidade Federal de Santa Catarina. Florianópolis, Santa Catarina, Brasil. https://orcid.org/00000003-0032-679

4 Enfermeiro. Especialista em Enfermagem de Urgência e Emergência. Universidade Federal de Santa Catarina. Florianópolis, Santa Catarina, Brasil. https://orcid. org/0000-0002-8840-4653

5 Enfermeira. Doutora em Filosofia da Enfermagem. Vice-Reitora da Universidade Federal de Santa Catarina. Florianópolis, Santa Catarina, Brasil. https://orcid. org/0000-0003-4845-8515
} 
emotional exhaustion, depersonalization and personal fulfillment. No significant differences were identified among the burnout mean values and the socio-professional characteristics of the participants, or among the hospital sectors.

Keywords: Professional Exhaustion. Job Satisfaction. Psychological Stress. Nursing.

Objetivo: identificar los niveles del sindrome de burnout entre enfermeros de un hospital universitario. Método: estudio transversal, realizado con 106 enfermeros en un hospital universitario de la Región Sur de Brasil. Los datos se recolectaron por medio de un cuestionario compuesto por dos instrumentos autorrespondidos: ficha de caracterización personal e Inventario de Burnout de Maslach. Resultados: los enfermeros que participaron de la investigación presentaron niveles moderados de burnout para las tres subescalas: agotamiento emocional $(21,9 \pm 5,0)$, despersonalización $(8,1 \pm 2,6)$ y realización personal $(30,4 \pm 3,2)$. Los enfermeros del centro quirúrgico presentaron un indice elevado en la subescala de agotamiento emocional. Conclusión: se identificó un nivel moderado de burnout entre los enfermeros de un hospital universitario para las tres subescalas: agotamiento emocional, despersonalización y realización personal. No se identificaron diferencias significativas entre las medias de burnout y las características socioprofisionales de los participantes, como asi tampoco entre los sectores hospitalarios.

Descriptores: Agotamiento Profesional. Satisfacción con el Empleo. Estrés Psicológico. Enfermería.

\section{Introdução}

O trabalho é um elemento importante no cotidiano da vida humana, pois, além de prover subsistência ao indivíduo, também gera sentimentos positivos relacionados ao alcance de realização profissional e crescimento pessoal. No entanto, quando o trabalho é desenvolvido em condições inadequadas, podem surgir repercussões negativas à saúde física e psíquica do trabalhador. Entre essas manifestações, um item de extrema importância é o estresse ocupacional, que leva à insatisfação profissional, à queda de rendimento no trabalho, ao absenteísmo e às doenças ocupacionais ${ }^{(1-3)}$.

Quando o estresse torna-se excessivo e crônico em decorrência da sobrecarga de trabalho, tem-se a síndrome de burnout. Essa síndrome tem adquirido cada vez mais evidência em estudos contemporâneos, principalmente entre aqueles que investigam a saúde do trabalhador de enfermagem. Essa profissão é potencialmente suscetível às interferências do contexto laboral que sugerem o esgotamento profissional, como a organização do trabalho em turnos, o contato diário com a morte e o sofrimento humano ${ }^{(3-5)}$.

Também designada como Síndrome do Esgotamento Profissional, essa doença caracteriza-se como o processo em que aspectos do contexto de trabalho e relacionamento interpessoal contribuem para o desenvolvimento de sofrimento psíquico relacionado ao contexto laboral ${ }^{(4-5)}$. Ela costuma iniciar com uma sensação de desgaste emocional, que é a primeira resposta ao estresse laboral crônico. Na sequência, tem-se a despersonalização, que se refere à percepção de deterioração da competência para resolver problemas e da satisfação com o trabalho. Como consequência, observam-se manifestações como ansiedade, irritabilidade, desmotivação, descomprometimento e alienação. Além disso, o trabalhador também tende a se autoavaliar negativamente, tornando-se infeliz e insatisfeito com a sua atividade profissional ${ }^{(6-9)}$.

Desse modo, a atuação do trabalhador que está estressado na realização da sua atividade influencia a saúde do próprio profissional e compromete o seu desempenho na vida pessoal, social e ocupacional, podendo repercutir, ainda, na assistência prestada aos pacientes, quando a ocupação é executada nos serviços de saúde. Assim, fatores presentes no ambiente de trabalho, capazes de promover insatisfação e sofrimento, além de interferirem negativamente na qualidade de vida dos trabalhadores, devem ganhar uma atenção diferenciada pelos gestores de saúde. Devido às repercussões individuais e coletivas supracitadas, a síndrome de burnout tem despertado interesse e preocupação de 
pesquisadores e organizações governamentais, empresariais e sindicais ${ }^{(5-9)}$.

$\mathrm{Na}$ literatura científica da Enfermagem, foram identificadas pesquisas realizadas sobre a síndrome de burnout entre enfermeiros no contexto hospitalar ${ }^{(5,8-11)}$ e pré-hospitalar ${ }^{(7)}$, que indicaram a necessidade de estudos contínuos sobre esse tema, para divulgar e auxiliar na diminuição dos seus efeitos entre os profissionais. Dessa forma, este estudo representa uma contribuição para a construção do conhecimento sobre saúde do trabalhador e a discussão sobre síndrome de burnout entre enfermeiros hospitalares. Diante das considerações apresentadas, estabeleceu-se a seguinte questão de pesquisa: Qual é a prevalência da síndrome de burnout em enfermeiros de um hospital universitário?

O objetivo deste estudo foi identificar os níveis da síndrome de burnout entre enfermeiros de um hospital universitário.

\section{Método}

Trata-se de um estudo transversal, desenvolvido em um hospital universitário público da Região Sul do Brasil, o qual dispõe de 268 leitos. O serviço de enfermagem da instituição está organizado e estruturado em quatro departamentos assistenciais: Emergência e Ambulatório, Clínica Médica, Clínica Cirúrgica e Saúde da Mulher, Criança e Adolescente.

A população elegível para a pesquisa foi constituída de 162 enfermeiros vinculados à Direção de Enfermagem da instituição. Os critérios de inclusão foram: exercício de atividades assistenciais e tempo de experiência igual ou superior a três meses no atual local de trabalho. O período de três meses foi definido com base na premissa de que esse tempo é o mínimo necessário para ambientação de um profissional em um novo local de trabalho. Excluíram-se os participantes ausentes por motivo de férias ou licença de qualquer natureza.

Assim, dos 162 enfermeiros do hospital, 12 desempenhavam cargos gerenciais, 2 não desempenhavam atividades assistenciais nos seus setores de atuação, 7 possuíam menos de 3 meses de atuação no atual local de trabalho e 9 encontravam-se de férias ou licença para tratamento de saúde. Dentre os 132 enfermeiros que atenderam os critérios de inclusão e exclusão, 9 negaram-se a participar da pesquisa e 17 não devolveram os instrumentos. Ao considerar o número de enfermeiros aptos a participar do estudo, obtiveram-se 106 (80,3\%) participantes, o que corresponde à amostra intencional do estudo obtida com a população acessada.

A coleta de dados foi realizada de novembro de 2012 a novembro de 2013, por meio de dois instrumentos autorrespondidos: ficha de caracterização pessoal e profissional e Inventário de Burnout de Maslach (IBM) ${ }^{(12)}$. A ficha de caracterização pessoal e profissional abordou características pessoais (idade, sexo e estado civil) e características profissionais (formação profissional, tempo de experiência na profissão, turno de trabalho, tempo de trabalho na unidade e na instituição, carga horária semanal e existência de outro vínculo empregatício). O IBM foi adaptado e validado para a cultura brasileira e tem por objetivo mensurar o desgaste profissional por meio da avaliação do sentimento do indivíduo em relação ao seu trabalho. Trata-se de um instrumento autoaplicável, que contém 22 afirmações relacionadas aos sentimentos pelo trabalho.

A avaliação da síndrome de burnout ocorre em três dimensões independentes, mas relacionadas entre si: exaustão emocional, despersonalização e diminuição da realização pessoal. A exaustão emocional corresponde ao desgaste físico e esgotamento emocional para lidar com situações estressoras. A despersonalização é a tendência do trabalhador de se autoavaliar de forma negativa, tornando-se infeliz e insatisfeito com o seu desenvolvimento profissional. A diminuição da realização pessoal envolve o desenvolvimento de atitudes frias, negativas e insensíveis direcionadas aos receptores de um serviço prestado $^{(13)}$. Neste estudo, o Alfa de Cronbach das subescalas exaustão emocional, despersonalização e diminuição da realização pessoal foi, respectivamente, 0,85, 0,69 e 0,60.

Os itens devem ser pontuados de acordo com a frequência da vivência de determinadas 
situações pelo profissional: (1) nunca, (2) algumas vezes no ano, (3) algumas vezes ao mês, (4) algumas vezes na semana e (5) diariamente $^{(12)}$. Para avaliação dos resultados, deve-se obter a soma das respostas dos participantes para cada subescala, que pode variar entre $9 \mathrm{e}$ 45 pontos para a subescala exaustão emocional, entre 5 e 25 para a subescala despersonalização e entre 8 e 40 para a subescala diminuição da realização pessoal. É importante ressaltar que, nas subescalas exaustão emocional e despersonalização, quanto maior a pontuação, maiores os sentimentos de exaustão emocional e de despersonalização percebidos pelo enfermeiro. $\mathrm{Na}$ subescala diminuição da realização pessoal, por possuir um escore inverso ao das outras subescalas, maiores pontuações indicam alta realização pessoal $^{(11)}$.

Para análise do nível da síndrome de burnout entre os enfermeiros, obteve-se a somatória referente à exaustão emocional, despersonalização e diminuição da realização pessoal. Em seguida, realizou-se o cálculo de corte dos percentis para estabelecer os níveis baixo, moderado e alto, cujo intervalo de pontuação encontra-se na Tabela 1.

Tabela 1 - Classificação dos níveis de burnout, segundo os percentis para cada subescala. Florianópolis, Santa Catarina, Brasil - 2012-2013

\begin{tabular}{l|c|c|c}
\hline \multirow{2}{*}{ Subescala } & \multicolumn{3}{|c}{ Burnout } \\
\cline { 2 - 4 } & Baixo & Moderado & Alto \\
\hline Exaustão emocional & $\leq 18$ & $19-24$ & $\geq 25$ \\
Despersonalização & $\leq 6$ & $7-9$ & $\geq 10$ \\
Diminuição da realização & $\geq 32$ & $28-31$ & $\leq 28$ \\
pessoal & & & \\
\hline
\end{tabular}

Fonte: Elaboração própria,

Os dados coletados foram tabulados no Excel® 2013 e analisados no programa Statistical Package for the Social Sciences (SPSS) para Windows, versão 19.0. Para descrição do perfil da amostra, utilizou-se estatística descritiva com elaboração de tabelas de frequência absoluta e relativa das variáveis categóricas e cálculo de medidas de posição e dispersão (média, mediana, desvio padrão, valores mínimo e máximo) para as variáveis contínuas. Para a comparação entre grupos, foi utilizada a análise de variância (ANOVA) e o Teste de Bonferroni. Os testes estatísticos foram considerados significativos quando p-valor $<0,05$.

Para atender aos aspectos éticos, o estudo foi aprovado pelo Comitê de Ética em Pesquisa de referência, mediante Parecer no 144.436 e CAAE: 09885612.1.0000.0121, e seguiu as orientações da Resolução n. 466/2012 do Conselho Nacional de Saúde. Todos os integrantes da pesquisa foram esclarecidos sobre os objetivos e a metodologia propostos, bem como tiveram assegurado seu direito de acesso aos dados. O consentimento livre e esclarecido por escrito foi solicitado, garantindo a confidencialidade da identidade dos participantes e das informações colhidas.

\section{Resultados}

A amostra foi composta por 106 enfermeiros, sendo Emergência (17,9\%), Clínica Médica (14,2\%) e Unidade de Terapia Intensiva (13,2\%) as unidades com maior número de participantes. Quanto ao turno de trabalho, prevaleceu o período noturno (32,1\%). A carga horária média de trabalho foi 37,41 (Desvio-Padrão=dp $\pm 11,80$ ). O tempo médio de experiência profissional foi de 13,5 anos $(d p \pm 9,46)$ e o tempo médio de trabalho na instituição foi de 12 anos $(\mathrm{dp} \pm 9,69)$. As demais variáveis de caracterização pessoal e profissional estão apresentadas na Tabela 2. 
Tabela 2 - Caracterização dos participantes do estudo. Florianópolis, Santa Catarina, Brasil - 2012-2013 $(n=106)$

\begin{tabular}{|c|c|c|}
\hline Variáveis & $\mathbf{n}$ & $\%$ \\
\hline \multicolumn{3}{|l|}{ Idade (anos) } \\
\hline 30 ou menos & 29 & 27,4 \\
\hline 31 a 40 & 33 & 31,1 \\
\hline 41 a 50 & 32 & 30,2 \\
\hline 51 ou mais & 12 & 11,3 \\
\hline \multicolumn{3}{|l|}{ Sexo } \\
\hline Feminino & 98 & 92,5 \\
\hline Masculino & 8 & 7,5 \\
\hline \multicolumn{3}{|l|}{ Estado civil } \\
\hline Casado & 53 & 50,0 \\
\hline Solteiro & 35 & 33,0 \\
\hline Separado & 10 & 9,4 \\
\hline Não respondeu & 8 & 7,5 \\
\hline \multicolumn{3}{|l|}{ Formação profissional } \\
\hline Especialização (lato sensu) & 49 & 46,2 \\
\hline Mestrado & 41 & 38,7 \\
\hline Graduação & 10 & 9,4 \\
\hline Doutorado & 6 & 5,7 \\
\hline \multicolumn{3}{|l|}{ Outro vínculo empregatício } \\
\hline Não & 89 & 84,0 \\
\hline Sim & 17 & 16 \\
\hline
\end{tabular}

Fonte: Elaboração própria.

Na Tabela 3, estão apresentados as médias e os desvios padrão das subescalas do IBM, segundo cada setor hospitalar. Conforme a classificação dos níveis de burnout segundo os percentis, os participantes da pesquisa apresentaram, no geral, níveis moderados para as três subescalas analisadas. A comparação entre as médias de burnout e as características socioprofissionais dos participantes não gerou diferenças estatisticamente significativas.

Tabela 3-Média e desvio-padrão do IBM, conforme setores hospitalares. Florianópolis, Santa Catarina, Brasil - 2012-2013 ( $n=106)$

(continua)

\begin{tabular}{|c|c|c|c|c|c|c|}
\hline \multirow[t]{2}{*}{ Variáveis } & \multicolumn{2}{|c|}{$\begin{array}{c}\text { Exaustão } \\
\text { emocional }\end{array}$} & \multicolumn{2}{|c|}{ Despersonalização } & \multicolumn{2}{|c|}{$\begin{array}{l}\text { Diminuição da } \\
\text { realização pessoal }\end{array}$} \\
\hline & Média & DP* & Média & DP* & Média & DP* \\
\hline Alojamento conjunto & 18,2 & 2,6 & 6,2 & 1,3 & 31,7 & 3,2 \\
\hline Ambulatório & 20,0 & 5,4 & 6,8 & 2,7 & 32,7 & 2,5 \\
\hline Centro cirúrgico & 27,0 & 7,0 & 9,0 & 0 & 30,5 & 0,7 \\
\hline Clínica cirúrgica & 24,3 & 4,7 & 9,9 & 2,4 & 28,5 & 2,8 \\
\hline Clínica médica & 20,6 & 5,2 & 7,2 & 2,4 & 31,7 & 2,8 \\
\hline Centro obstétrico & 22,2 & 4,9 & 5,8 & 0,9 & 28,6 & 4,9 \\
\hline Emergência & 23,0 & 5,1 & 9,3 & 2,9 & 30,2 & 3,6 \\
\hline
\end{tabular}


Tabela 3-Média e desvio-padrão do IBM, conforme setores hospitalares. Florianópolis, Santa Catarina, Brasil - 2012-2013 ( $n=106)$

(conclusão)

\begin{tabular}{l|c|c|c|c|c|c}
\hline \multirow{2}{*}{ Variáveis } & \multicolumn{2}{|c|}{$\begin{array}{c}\text { Exaustão } \\
\text { emocional }\end{array}$} & \multicolumn{2}{c|}{ Despersonalização } & \multicolumn{2}{c}{$\begin{array}{c}\text { Diminuição da } \\
\text { realização pessoal }\end{array}$} \\
\cline { 2 - 7 } & Média & $\mathbf{D P}$ & Média & DP* & Média & DP* $^{*}$ \\
\hline Hemodiálise & 22,6 & 6,6 & 9,3 & 3,2 & 28,6 & 3,5 \\
Pediatria & 19,1 & 5,9 & 7,5 & 1,8 & 32,3 & 3,0 \\
Unidade ginecológica & 21,6 & 5,9 & 8,5 & 4,3 & 30,7 & 2,7 \\
Unidade de Terapia Intensiva & 22,8 & 4,2 & 8,5 & 1,8 & 29,4 & 2,4 \\
Unidade de Terapia Intensiva & 22,6 & 4,2 & 7,6 & 1,6 & 29,8 & 2,6 \\
Neonatal & & & & & & $\mathbf{3 0 , 4}$ \\
Total & $\mathbf{2 1 , 9}$ & $\mathbf{5 , 0}$ & $\mathbf{8 , 1}$ & $\mathbf{2 , 6}$ & $\mathbf{3 0 , 4}$ \\
\hline
\end{tabular}

Fonte: Elaboração própria.

*DP = desvio padrão.

Não foram identificadas diferenças significativas entre os resultados das subescalas e os setores hospitalares. Entretanto, os enfermeiros do centro cirúrgico apresentaram índice alto na subescala exaustão emocional. Na subescala despersonalização, os enfermeiros de nenhum setor atingiram o escore alto e a menor média foi identificada no centro obstétrico. Na subescala diminuição da realização pessoal, os menores índices foram constatados na pediatria e no ambulatório.

\section{Discussão}

O grupo de enfermeiros participantes deste estudo foi constituído predominantemente de profissionais em meio de carreira e do sexo feminino. Esse resultado vai ao encontro do perfil da enfermagem no Brasil ${ }^{(14)}$ e de achados descritos por estudos desenvolvidos anteriormente ${ }^{(16-18)}$.

Quanto ao tempo de atuação profissional, identificou-se que as médias do tempo de experiência profissional e na instituição eram superiores em relação ao período de atuação no setor. Isso pode indicar a existência de alta rotatividade de enfermeiros entre os setores de trabalho. A relação entre insatisfação no trabalho e a alta rotatividade profissional foi encontrada em revisão de literatura sobre as consequências do sofrimento moral em enfermeiros. Essa associação pode ter como consequência o prejuízo da qualidade do cuidado ${ }^{(15)}$.

No tangente à formação, destaca-se a alta qualificação profissional dos enfermeiros, pois
96 (90,6\%) participantes eram pelo menos especialistas. Tal resultado assemelha-se ao de estudos anteriores, em que a maioria dos enfermeiros possuía algum tipo de pós-graduação ${ }^{(16-18)}$. Tal nível de formação profissional pode ser justificado pela vinculação acadêmica da instituição em que os dados foram coletados, pois hospitais universitários destacam-se pelo incentivo e pela valorização do desenvolvimento profissional dos trabalhadores.

Os resultados gerais do IBM para a subescala exaustão emocional foi 21,9 $( \pm 5,0)$, para despersonalização foi $8,1( \pm 2,6)$ e para diminuição da realização pessoal foi 30,4 $( \pm 3,2)$, o que indica nível moderado de burnout entre os participantes. Achados semelhantes também estão descritos em estudos anteriores realizados no Brasil ${ }^{(5,19)}$. Em comparação com os resultados de pesquisa multicêntrica realizada em oito países, constatou-se que a média de exaustão emocional obtida neste estudo foi menor em relação à de enfermeiros do Japão. O grau de despersonalização foi menor, na comparação com os resultados dos Estados Unidos e Japão. Em contrapartida, a realização pessoal foi menor, principalmente em relação aos Estados Unidos, Canadá e Nova Zelândia ${ }^{(9)}$.

$\mathrm{Na}$ comparação entre as características socioprofissionais e as médias de burnout dos participantes, não houve diferenças estatisticamente significativas. Isso pode ser justificado pelo tamanho da amostra e pela multiplicidade 
de aspectos que envolvem a variável em estudo. Além disso, a amostra do estudo foi composta, em sua grande maioria, pelo sexo feminino, o que dificultou o estabelecimento de padrões estatísticos e a análise dos dados comparativos entre os sexos.

Entretanto, estudo sobre a presença da Síndrome de burnout entre profissionais de Enfermagem das Unidades de Terapia Intensiva de um Hospital Universitário identificou maior prevalência entre participantes do sexo feminino, casados e adultos jovens. Além disso, maior carga horária mostrou significância positiva, com alto padrão de despersonalização e exaustão emocional ${ }^{(5)}$.

De acordo com a literatura, mulheres têm maior vulnerabilidade ao burnout, pois são mais propensas a se envolver com os problemas das pessoas que estão sob seus cuidados ${ }^{(20)}$. Em relação à idade, a idealização e expectativas elevadas são mais comuns entre trabalhadores jovens, o que pode predispor ao desenvolvimento da síndrome de burnout ${ }^{(19)}$.

Em relação aos setores hospitalares, o centro cirúrgico foi o ambiente de trabalho em que os enfermeiros apresentaram nível alto de exaustão emocional. Esse resultado pode justificar-se por se tratar de um ambiente de elevadas demandas emocionais, influenciadas pela complexidade dos procedimentos e seus riscos de complicação ${ }^{(21)}$. Além disso, por se tratar de um cenário com atuação de diversas categorias profissionais, a ocorrência de conflitos de relacionamento e problemas de comunicação são comuns, principalmente entre equipe médica e de enfermagem. Nesse contexto, o enfermeiro destaca-se pela atuação na mediação e resolução de conflitos, visando à integração entre os profissionais ${ }^{(22)}$, o que pode representar também uma fonte de estresse e desgaste profissional.

Em contrapartida, os resultados desta pesquisa indicaram baixo grau de despersonalização em enfermeiros do centro obstétrico e alta realização pessoal daqueles que trabalhavam no ambulatório e na pediatria. Esses resultados podem estar associados à identificação pessoal com o cenário de atuação, bom relacionamento com a equipe de trabalho, à qualificação profissional e à disponibilidade de rede de apoio fora do ambiente laboral, fatores que predispõem para maior satisfação com trabalho ${ }^{(2-3,23)}$.

Outras pesquisas realizadas sobre burnout entre trabalhadores de enfermagem em diferentes cenários estão disponíveis e foram identificadas na literatura. Pesquisa transversal realizada com enfermeiros e auxiliares de enfermagem militares, atuantes em unidade de cuidados intensivos e críticos de um hospital do Peru, identificou escores mais altos de exaustão emocional e despersonalização, e menores pontuações na diminuição da realização pessoal, o que implica em maior grau de desgaste ${ }^{(8)}$.

Estudo longitudinal e não randomizado, realizado com enfermeiros de 13 hospitais de diferentes unidades da comunidade de Valência, Espanha, com o objetivo de examinar a relação entre burnout e satisfação no trabalho, concluiu que há uma relação bidirecional e longitudinal entre burnout e satisfação no trabalho. No entanto, os efeitos da síndrome de burnout como antecedente de satisfação no trabalho são mais fortes do que a satisfação no trabalho como antecedente de burnout ${ }^{(10)}$.

Pesquisa transversal realizada com profissionais de um Serviço de Atendimento Móvel de Urgência (enfermeiros, técnicos de enfermagem, auxiliares de enfermagem, médicos e condutores) identificou escores altos para exaustão emocional e despersonalização. A exaustão emocional e a despersonalização foram maiores na equipe médica, quando comparada à equipe de enfermagem ${ }^{(7)}$. Estudo realizado com enfermeiros em hospitais do Reino Unido evidenciou relação entre as dimensões da síndrome de burnout e a segurança do cuidado percebida pelo paciente ${ }^{(24)}$. Além disso, conforme revisão sistemática da literatura, que analisou a associação entre bem-estar da equipe de saúde, burnout e segurança do paciente, níveis mais altos de burnout estão associados a maior frequência de erros diagnósticos ou terapêuticos, levando à baixa segurança do paciente ${ }^{(25)}$.

Diversas manifestações referentes às dimensões da síndrome de burnout, precisam de 
atenção e valorização por parte das instituições, com o planejamento e a implementação de medidas que favoreçam a redução do estresse no contexto laboral. O auxílio aos profissionais nos processos de enfrentamento de situações que parecem conduzi-los ao burnout constitui-se em uma possibilidade de contribuir para o fortalecimento do exercício do próprio cuidado ${ }^{(18)}$. Nesse sentido, destaca-se o papel dos gestores no provimento de condições favoráveis de trabalho para os profissionais, a fim de evitar níveis de esgotamento e/ou sofrimento psíquico-emocional, proporcionando melhorias na qualidade de vida dos trabalhadores, o que repercutirá em melhorias na qualidade assistencial.

O estudo apresenta como limitação representar a realidade de um único hospital da Região Sul do Brasil. Estudos com maior número de participantes e incluindo outros cenários hospitalares poderão gerar informações mais representativas do objeto investigado. Outra limitação refere-se ao delineamento transversal, pois, nesse, a causalidade reversa não pode ser descartada. É importante mencionar ainda as reconhecidas limitações da investigação sobre o burnout, devido à falta de consenso na literatura sobre se é necessário um ou três níveis altos das dimensões. Além disso, não há consenso acerca do ponto de corte utilizado na interpretação dos resultados do questionário, o que dificulta a verificação da prevalência de burnout e a comparação entre estudos.

\section{Conclusão}

Os enfermeiros participantes da pesquisa apresentaram níveis moderados de burnout para as três subescalas: exaustão emocional, despersonalização e realização pessoal. Não foram identificadas diferenças significativas entre as médias de burnout e as características socioprofissionais dos participantes, nem entre os setores hospitalares.
Recomenda-se que gestores da área de enfermagem e saúde no ambiente hospitalar desenvolvam ações e programas visando à saúde do trabalhador e a qualidade de vida no trabalho. Em relação a desdobramentos deste estudo, pontua-se a possibilidade de novas investigações que explorem a problemática em tela por meio de abordagem qualitativa, a fim de discutir aspectos que influenciam na satisfação profissional dos enfermeiros.

\section{Colaborações:}

1 - concepção, projeto, análise e interpretação dos dados: José Luís Guedes dos Santos, Caroline Cechinel Peiter e Alacoque Lorenzini Erdmann;

2 - redação do artigo e revisão crítica relevante do conteúdo intelectual: José Luís Guedes dos Santos, Rosângela Marion da Silva, Caroline Cechinel Peiter, Fernando Henrique Antunes Menegon e Alacoque Lorenzini Erdmann; 3 - aprovação final da versão a ser publicada: José Luís Guedes dos Santos, Rosângela Marion da Silva, Caroline Cechinel Peiter, Fernando Henrique Antunes Menegon e Alacoque Lorenzini Erdmann.

\section{Referências}

1. Bendassolli PF, Guedes SMG. Meaning, fulness and psychological function of work: discussing this conceptual triad and its methodological challenges. Av Psicol Latinoam [Internet]. 2014 Jan/Apr [cited 2018 Dec 22];32(1):131-47. Available from: https:// www.researchgate.net/publication/263179954_ Meaning_meaningfulness_and_psychological_ function_of_work_Discussing_this_conceptual_ triad_and_its_methodological_challenges

2. Miorin JD, Camponogara S, Pinno C, Beck CLC, Costa V, Freitas EO. Pleasure and pain of nursing workers at a first aid service. Texto contexto - enferm [Internet]. 2018 May [cited 2018 Dec 29];27(2):e2350015. Available from: http://www.scielo.br/scielo.php?script=sci arttext\&pid=S0104-07072018000200305\&lng=pt 
3. Michelin SR, Nitschke RG, Martini JG, Tholl AD, Souza LCSL, Henckemaier L. (Re)cognizing health center workers' routine: a path for burnout prevention and health promotion. Texto contexto - enferm [Internet]. 2018 [cited 2018 Dec 28];27(1):e5510015. Available from: http://www.scielo.br/scielo.php?script $=$ sci_ arttext\&pid=S0104-07072018000100305\&lng=en

4. Silva EA. O trabalho ameaçado. In: Benevides-Pereira AMT, organizador. Burnout: quando o trabalho ameaça o bem-estar do trabalhador. São Paulo: Casa do Psicólogo; 2002. p. 28-45.

5. Fernandes LS, Nitsche MJT, Godoy I. Association between burnout syndrome, harmful use of alcohol and smoking in nursing in the ICU of a university hospital. Ciênc Saúde Colet [Internet]. 2018 [cited 2018 Dec 28];23(1):203-14. Available from: http://www.scielo.br/pdf/csc/v23n1/en_14138123-csc-23-01-0203.pdf

6. França TLB, Oliveira ACBL, Lima LF, Melo JKF, Silva RAR. Burnout syndrome: characteristics, diagnosis, risk factors and prevention. Rev Enferm UFPE Online [Internet]. 2014 [cited 2018 Dec 28];8(10):3539-46. Available from: http://www. revista.ufpe.br/revistaenfermagem/index.php/ revista/index

7. Luz LM, Torres RRB, Sarmento KMVQ, Sales JMR, Farias KN, Marques MB. Burnout Syndrome in urgency mobile service professionals. Rev pesqui cuid fundam (online) [Internet]. 2017 [cited 2018 Dec 28];9(1):238-46. Available from: http://www. seer.unirio.br/index.php/cuidadofundamental/ article/view/5400/pdf

8. Ayala E, Camero AM. Determinants of burnout in acute and critical care military nursing personnel: a cross-sectional study from Peru. PLoS One [Internet]. 2013 [cited 2018 Dec 28];8(1):e54408. Available from: https://journals.plos.org/plosone/ article?id=10.1371/journal.pone .0054408

9. Poghosyan L, Aiken LH, Sloane DM. Factor structure of the Maslach burnout inventory: an analysis of data from large scale cross-sectional surveys of nurses from eight countries. Int J Nurs Stud [Internet]. 2009 Jul [cited 2018 Dec 28];46(7):894-902. Available from: https://www. ncbi.nlm.nih.gov/pubmed/19362309

10. Figueiredo-FerrazH, Grau-Alberola E, Gil-Monte PR, García-Juesas JA. Síndrome de quemarse por el trabajo y satisfacción laboral en profesionales de enfermería. Psicothema [Internet]. 2012 [cited 2018 Dec 28];24(2):271-6. Available from: http://www. psicothema.com/psicothema $\cdot$ asp? $\mathrm{id}=4010$

11. Gasparino RC, Guirardello EB. Professional practice environment and burnout among nurses. Rev Rene [Internet]. 2015 [cited 2018 Dec 28];16(1):90-6. Available from: www.revistarene.ufc.br/revista/ index.php/revista/article/viewFile/1862/pdf

12. Maslach C, Jackson SE. The measurement of experienced burnout. J Occup Behav [Internet]. 1981 [cited 2018 Dec 28];2(2):99-113. Available from: https://onlinelibrary.wiley.com/doi/abs/10.1002/ job.4030020205

13. Lautert L. The professional fatigue: empirical study with hospital nurses. Rev Gaúcha Enferm. [Internet]. 1997 [cited 2018 Dec 28];18(2):133-44. Available from: https://seer.ufrgs.br/RevistaGauchadeEnfermagem/ article/view/4140/42827

14. MachadoMH, AguiarFilhoW, Lacerda WF, OliveiraE, Lemos W, Wermelinger $\mathrm{M}$, et al. Características gerais da enfermagem: o perfil sócio demográfico. Enferm Foco [Internet]. 2016 [citado 2018 Dez 28];7(esp):9-14. Available from: http://revista. cofen.gov.br/index.php/enfermagem/article/ view/686/296

15. Ramos FRS, Barth PO, Schneider AM, Cabral AS, Reinaldo JS. Effects of moral distress on nurses: integrative literature review. Cogitare Enferm [Internet]. 2016 Apr/Jun [cited 2018 Dec 28];21(2):1-13. Available from: https://revistas.ufpr. br/cogitare/article/view/45247/28570

16. Panunto MR, Guirardello EB. Professional nursing practice: environment and emotional exhaustion among intensive care nurses. Rev Lat-Am Enfermagem [Internet]. 2013 Jun [cited 2018 Dec 28];21(3):765-72. Available from: http://www.scielo.br/pdf/rlae/v21n3/0104-1169rlae-21-03-0765.pdf

17. Morais BX, Pedro CMP, Dalmolin GL, Silva AM. Professional satisfaction of nursing workers from a service of hematology-oncology. Rev Rene [Internet]. 2018 [cited 2018 Dec 28];19:e3165. Available from: http://periodicos.ufc.br/rene/ article/download/31317/71755

18. Barth PO, Ramos FRS, Barlem ELD, Dalmolin GL, Schneider DG. Validation of a moral distress instrument in nurses of primary health care. Rev Latino-Am Enfermagem. 2018;26:e3010. DOI: http://dx.doi.org/10.1590/1518-8345.2227.3010 
19. Merces MC, Carneiro e Cordeiro TMS, Santana AIC, Lua I, Souza e Silva D, Alves MS, et al. Burnout syndrome in nursing workers of the primary health care. Rev baiana enferm [Internet]. 2016 [cited 2018 Dec 28];30(3):1-9. Available from: https://portalseer.ufba.br/index.php/enfermagem/ article/view/15645/pdf_64

20. Vasconcelos EM, Martino MMF. Predictors of burnout syndrome in intensive care nurses. Rev Gaúcha Enferm [Internet]. 2017 [cited 2018 Dec 29];38(4):e65354. Available from: http://www. scielo.br/scielo.php?script=sci_arttext\&pid=S1983$14472017000400417 \& \operatorname{lng}=\mathrm{en}$

21. Ferreira NN, Lucca SR. Burnout syndrome in nursing assistants of a public hospital in the state of São Paulo. Rev bras epidemiol [Internet]. 2015 [cited 2018 Dec 28];18(1):68-79. Available from: http://www.scielo.br/scielo.php?script $=$ sci_ arttext\&pid=S1415-790X2015000100068\&lng=en

22. Ingvarsdottir E, Halldorsdottir S. Enhancing patient safety in the operating theatre: from the perspective of experienced operating theatre nurses. Scand J Caring Sci [Internet]. 2018 [cited 2018 Dec 30];32(2):951-60. Available from: https:// www.ncbi.nlm.nih.gov/pubmed/28940247
23. Silva DKC, Pacheco MJT, Marques HS, Castelo Branco RC, Silva MACN, Nascimento MDSB. Burnout no trabalho de médicos pediatras. Rev Bras Med Trab [Internet]. 2017 [cited 2018 Dec 30];15(1):2-11. Available from: http://pesquisa. bvsalud.org/portal/resource/pt/biblio-833203

24. Johnson J, Louch G, Dunning A, Johnson O, Grange A, Reynolds C, et al. Burnout mediates the association between depression and patient safety perceptions: a cross-sectional study in hospital nurses. J Adv Nurs [internet]. 2017 Jul [cited 2018 Dec 30];73(7):1667-80. Available from: https:// www.ncbi.nlm.nih.gov/pubmed/28072469

25. Hall LH, Johnson J, Watt I, Tsipa A, O'Connor DB. Healthcare Staff Wellbeing, Burnout, and Patient Safety: A Systematic Review. PLoS One [Internet]. 2016 Jul [cited 2018 Dec 30];11(7):e0159015. Available from: https://journals.plos.org/plosone/ article?id=10.1371/journal.pone.0159015

Recebido: 31 de dezembro de 2018

Aprovado: 23 de setembro de 2019

Publicado: 23 de janeiro de 2020

A Revista Baiana de Enfermagem utiliza a Licença Creative Commons - Atribuição-NãoComercial 4.0 Internacional.

https://creativecommons.org/licenses/by-nc/4.0/

Este artigo é de acesso aberto distribuído sob os termos da Licença Creative Commons (CC BY-NC).

Esta licença permite que outros remixem, adaptem e criem a partir do seu trabalho para fins não comerciais. Embora os novos trabalhos tenham de lhe atribuir o devido crédito e não possam ser usados para fins comerciais, os usuários não têm de licenciar esses trabalhos derivados sob os mesmos termos. 